\title{
Combinatorial Proof of the Minimal Excludant Theorem
}

\author{
Cristina Ballantine \\ Department of Mathematics and Computer Science \\ College of The Holy Cross \\ Worcester, MA 01610, USA \\ cballant@holycross.edu \\ Mircea Merca \\ Academy of Romanian Scientists \\ Ilfov 3, Sector 5, Bucharest, Romania \\ mircea.merca@profinfo.edu.ro
}

\begin{abstract}
The minimal excludant of a partition $\lambda, \operatorname{mex}(\lambda)$, is the smallest positive integer that is not a part of $\lambda$. For a positive integer $n, \sigma \operatorname{mex}(n)$ denotes the sum of the minimal excludants of all partitions of $n$. Recently, Andrews and Newman obtained a new combinatorial interpretations for $\sigma \operatorname{mex}(n)$. They showed, using generating functions, that $\sigma \operatorname{mex}(n)$ equals the number of partitions of $n$ into distinct parts using two colors. In this paper, we provide a purely combinatorial proof of this result and new properties of the function $\sigma \operatorname{mex}(n)$. We generalize this combinatorial interpretation to $\sigma_{r} \operatorname{mex}(n)$, the sum of least $r$-gaps in all partitions of $n$. The least $r$-gap of a partition $\lambda$ is the smallest positive integer that does not appear at least $r$ times as a part of $\lambda$.
\end{abstract}

Keywords: Minimal excludant, MEX, least gap in partition, partitions, 2-color partitions

MSC 2010: 11A63, 11P81, 05A19

\section{Introduction}

The minimal excludant or mex-function on a set $S$ of positive integers is the least positive integer not in $S$. The history of this notion goes back to at least the 1930s when it was applied to combinatorial game theory [11, 9].

Recently, Andrews and Newman [4] considered the mex-function applied to integer partitions. They defined the minimal excludant of a partition $\lambda, \operatorname{mex}(\lambda)$, 
to be the smallest positive integer that is not a part of $\lambda$. In addition, for each positive integer $n$, they defined

$$
\sigma \operatorname{mex}(n):=\sum_{\lambda \in \mathcal{P}(n)} \operatorname{mex}(\lambda)
$$

where $\mathcal{P}(n)$ is the set of all partitions of $n$. Elsewhere in the literature, the minimal excludant of a partition $\lambda$ is referred to as the least gap or smallest gap of $\lambda$. An exact and asymptotic formula for $\sigma \operatorname{mex}(n)$ is given in [8]. In [5], where $\operatorname{mex}(\lambda)$ is denoted by $g_{1}(\lambda)$ and $\sigma \operatorname{mex}(n)$ is denoted by $S_{1}(n)$, the authors study a generalization of $\sigma \operatorname{mex}(n)$ and its connection to polygonal numbers.

Let $\mathcal{D}_{2}(n)$ be the set of partitions of $n$ into distinct parts using two colors and let $D_{2}(n)=\left|\mathcal{D}_{2}(n)\right|$. For ease of notation, we denote the colors of the parts of partitions in $\mathcal{D}_{2}(n)$ by 0 and 1. In [4], the authors give two proofs of the following theorem.

Theorem 1.1. Given an integer $n \geqslant 0$, we have

$$
\sigma \operatorname{mex}(n)=D_{2}(n)
$$

They also determine the parity of the $\sigma$ mex function.

Lemma 1.2. $\sigma \operatorname{mex}(n)$ is odd if and only if $n=j(3 j \pm 1)$ for some non-negative integer $j$.

We note that this parity result was also established in [5, Corollary 1.6]. Andrews and Newman write that "it would be of great interest to have a bijective proof of Theorem 1.1." They also ask for a combinatorial proof of Lemma 1.2. In section 2 we provide these desired proofs. In the proof of Theorem 1.1, we make use of the fact that

$$
\sigma \operatorname{mex}(n)=\sum_{k \geqslant 0} p(n-k(k+1) / 2),
$$

where, as usual, $p(n)$ denotes the number of partitions of $n$. A combinatorial proof of (1) is given in [5, Theorem 1.1] . The same argument is also described in the second proof of [4, Theorem 1.1]. We note the result proven in [5] is a generalization of (1) to the sum of $r$-gaps in all partitions of $n$. The $r$-gap of a partition $\lambda$ is the least positive integer that does not appear $r$ times as a part of $\lambda$.

In [2], Andrews and Merca considered a restricted mex-function and defined $M_{k}(n)$ to be the number of partitions of $n$ in which $k$ is the least positive integer that is not a part and there are more parts $>k$ than there are parts $<k$.

When $k=1, M_{1}(0)=0$ and, if $n>0, M_{1}(n)$ is the number of partitions of $n$ that do not contain 1 as a part. Thus, if $n>0$, we have $M_{1}(n)=p(n)-p(n-1)$. Then, from (1), we obtain

$$
\sigma \operatorname{mex}(n)-\sigma \operatorname{mex}(n-1)-\delta(n)=\sum_{j=0}^{\infty} M_{1}(n-j(j+1) / 2),
$$


where $\delta$ is the characteristic function of the set of triangular numbers, i.e.,

$$
\delta(n)= \begin{cases}1, & \text { if } n=m(m+1) / 2, m \in \mathbb{Z}, \\ 0, & \text { otherwise. }\end{cases}
$$

In section 3, we prove the following generalization of (2).

Theorem 1.3. Let $k$ be a positive integer. Given an integer $n \geqslant 0$, we have

$$
\begin{aligned}
& (-1)^{k-1}\left(\sum_{j=-(k-1)}^{k}(-1)^{j} \sigma \operatorname{mex}(n-j(3 j-1) / 2)-\delta(n)\right) \\
& =\sum_{j=0}^{\infty} M_{k}(n-j(j+1) / 2) .
\end{aligned}
$$

As a corollary of this theorem we obtain the following infinite family of linear inequalities involving $\sigma$ mex.

Corollary 1.4. Let $k$ be a positive integer. Given an integer $n \geqslant 0$, we have

$$
(-1)^{k-1}\left(\sum_{j=-(k-1)}^{k}(-1)^{j} \sigma \operatorname{mex}(n-j(3 j-1) / 2)-\delta(n)\right) \geqslant 0
$$

with strict inequality if $n \geqslant k(3 k+1) / 2$.

In section 3 we also give a combinatorial interpretation for

$$
\sum_{j=0}^{\infty} M_{k}(n-j(j+1) / 2)
$$

in terms of the number of partitions into distinct parts using three colors and satisfying certain conditions.

In sections 4 and 5, we introduce connections to certain subsets of overpartitions and partitions with distinct parts, respectively.

\section{Combinatorial Proofs of Theorem 1.1 and Lemma 1.2}

\subsection{Bijective Proof of Theorem 1.1}

To prove the theorem, we adapt Sylvester's bijective proof of Jacobi's triple product identity [12] which was later rediscovered by Wright [13]. For the interested reader, it is probably easier to follow Wright's short article for the description of the bijection.

Given a partition $\lambda$ in $\mathcal{D}_{2}(n)$, let $\lambda^{(j)}, j=0,1$, be the (uncolored) partition whose parts are the parts of color $j$ in $\lambda$. Then, $\lambda^{(1)}$ and $\lambda^{(2)}$ are partitions into distinct parts. 
Example 2.1. If $\lambda=4_{1}+3_{0}+3_{1}+2_{0}+1_{0} \in \mathcal{D}_{2}(13)$, then $\lambda^{(0)}=3+2+1$ and $\lambda^{(1)}=4+3$.

Denote by $\eta(k)$ the staircase partition $\eta(k)=k+(k-1)+(k-2)+\cdots+3+2+1$. If $k=0$ we define $\eta(k)=\emptyset$. For any partition $\lambda$ we denote by $\ell(\lambda)$ the number of parts in $\lambda$.

Definition 1. Given diagram of left justified rows of boxes (not necessarily the Ferrers diagram of a partition), the staircase profile of the diagram is a zig-zag line starting in the upper left corner of the diagram with a right step and continuing in alternating down and right steps until the end of a row of the diagram is reached.

Example 2.2. The staircase profile of the diagram



is

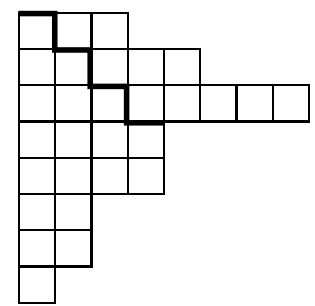

Given a Ferrers diagram (with boxes of unit length) of a partition $\lambda$ into distinct parts, the shifted Ferrers diagram of $\lambda$ is the diagram in which row $i$ is shifted $i-1$ units to the right.

We create a map

$$
\varphi: \bigcup_{k \geqslant 0} \mathcal{P}(n-k(k+1) / 2) \rightarrow \mathcal{D}_{2}(n)
$$

as follows. Start with $\lambda \in \mathcal{P}(n-k(k+1) / 2)$ for some $k \geqslant 0$. Append a diagram with rows of lengths $1,2, \ldots k$ (i.e., the Ferrers diagram of $\eta(k)$ rotated by $180^{\circ}$ ) the top of Ferrers diagram of $\lambda$. We obtain a diagram with $n$ boxes. Draw the staircase profile of the new diagram. Let $\alpha$ be the partition whose parts are the length of the columns to the left of the staircase profile and let $\beta$ be the partition whose parts are the length of the rows to the right of the staircase profile. Then $\alpha$ and $\beta$ are partitions with distinct parts. Moreover, $k \leqslant \ell(\alpha)-\ell(\beta) \leqslant k+1$. 
Color the parts of $\alpha$ with color $k(\bmod 2)$ and the parts of $\beta$ with color $k+1$ $(\bmod 2)$. Then $\varphi(\lambda)$ is defined as the 2 -color partition of $n$ whose parts are the colored parts of $\alpha$ and $\beta$.

Conversely, start with $\mu \in \mathcal{D}_{2}(n)$. Let $\ell_{j}(\mu), j=0,1$, be the number of parts of color $j$ in $\mu$.

(i) If $\ell_{0}(\mu) \geqslant \ell_{1}(\mu)$, let $r=\ell_{0}(\mu)-\ell_{1}(\mu)$. Let $k=r+\frac{(-1)^{r}-1}{2}$. Remove the top $k$ rows (i.e., the rotated Ferrers diagram of $\eta(k))$ from the conjugate of the shifted diagram of $\mu^{(0)}$ and join the remaining diagram with the shifted digram of $\mu^{(1)}$ so they align at the top. The obtained partition $\varphi^{-1}(\mu)$ belongs to $\mathcal{P}(n-k(k+1) / 2)$.

(ii) If $\ell_{1}(\mu)>\ell_{0}(\mu)$, let $r=\ell_{1}(\mu)-\ell_{0}(\mu)$. Let $k=r-\frac{(-1)^{r}+1}{2}$. Remove the top $k$ rows (i.e., the rotated Ferrers diagram of $\eta(k)$ ) from the conjugate of the shifted diagram of $\mu^{(1)}$ and join the remaining diagram with the shifted digram of $\mu^{(0)}$ so they align at the top. The obtained partition $\varphi^{-1}(\mu)$ belongs to $\mathcal{P}(n-k(k+1) / 2)$.

Example 2.3. Let $n=38, k=3$, and let $\lambda=7+7+6+6+4+2$ be a partition of $n-k(k+1) / 2=32$. We add the rotated Ferrers diagram of $\eta(3)$ to the top of the Ferrers diagram of $\lambda$ and draw the staircase profile.

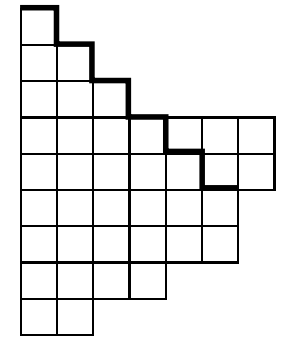

Then $\alpha=9+8+6+5+3+2$ and $\beta=3+2$. Since $k$ is odd, we have $\varphi(\lambda)=9_{1}+8_{1}+6_{1}+5_{1}+3_{1}+3_{0}+2_{1}+2_{0} \in \mathcal{D}_{2}(38)$.

Conversely, suppose $\mu=9_{1}+8_{1}+6_{1}+5_{1}+3_{1}+3_{0}+2_{1}+2_{0} \in \mathcal{D}_{2}(38)$. Then $\ell_{0}(\mu)=2$ and $\ell_{1}(\mu)=6$. We have $r=\ell_{1}(\mu)-\ell_{0}(\mu)=4$ and $k=3$. The diagrams of the conjugate of the shifted diagram of $\mu^{(1)}$ and the shifted diagram of $\mu^{(0)}$ are shown below.

Next, we remove the first 3 rows from the conjugate of the shifted diagram of $\mu^{(1)}$ and join the remaining diagram and the shifted digram of $\mu^{(0)}$ so they align at the top. We obtain $\varphi^{-1}(\mu)=7+7+6+6+4+2 \in \mathcal{P}(32)$.

\subsection{Combinatorial Proof of Lemma 1.2}

To determine the parity of $\sigma \operatorname{mex}(n)$, we pair partitions in $\mathcal{D}_{2}(n)$ as follows. If $\mu \in \mathcal{D}_{2}(n)$, we denote by $\tilde{\mu}$ the partition in $\mathcal{D}_{2}(n)$ obtained by interchanging the colors of the part of $\mu$. Then $\mu \neq \tilde{\mu}$ if and only if $\mu^{(0)} \neq \mu^{(1)}$. If $n$ is odd, $\mu^{(0)} \neq \mu^{(1)}$ for all $\mu \in \mathcal{D}_{2}(n)$ and $\sigma$ mex is even. If $n$ is even, $\sigma$ mex $\equiv q(n / 2)$ $(\bmod 2)$, where, as usual, $q(m)$ denotes the number of partitions of $m$ with distinct parts. Franklin's involution used to prove Euler's Pentagonal Number Theorem provides a pairing of partitions with distinct parts that shows that $q(m)$ is odd if and only if $m$ is a generalized pentagonal number. Thus, $\sigma \operatorname{mex}(n)$ is odd if and only if $n$ is twice a generalized pentagonal number.

\section{Proofs of Theorem $\mathbf{1 . 3}$}

Analytic proof of Theorem 1.3. In [2], the authors considered Euler's pentagonal number theorem and proved the following truncated form:

$$
\frac{(-1)^{k-1}}{(q ; q)_{\infty}} \sum_{n=-(k-1)}^{k}(-1)^{j} q^{n(3 n-1) / 2}=(-1)^{k-1}+\sum_{n=k}^{\infty} \frac{q^{\left(\begin{array}{c}
k \\
2
\end{array}\right)+(k+1) n}}{(q ; q)_{n}}\left[\begin{array}{l}
n-1 \\
k-1
\end{array}\right],
$$

where

$$
(a ; q)_{n}= \begin{cases}1, & \text { if } n=0 \\ \prod_{k=0}^{n-1}\left(1-a q^{k}\right), & \text { otherwise }\end{cases}
$$

and

$$
\left[\begin{array}{l}
n \\
k
\end{array}\right]= \begin{cases}\frac{(q ; q)_{n}}{(q ; q)_{k}(q ; q)_{n-k}}, & \text { if } 0 \leqslant k \leqslant n \\
0, & \text { otherwise. }\end{cases}
$$

Multiplying both sides of (3) by

$$
\frac{\left(q^{2} ; q^{2}\right)_{\infty}}{\left(q, q^{2}\right)_{\infty}}=\sum_{n=0}^{\infty} q^{n(n+1) / 2},
$$

we obtain

$$
\begin{aligned}
& (-1)^{k-1}\left(\left(\sum_{n=0}^{\infty} \sigma \operatorname{mex}(n) q^{n}\right)\left(\sum_{n=-(k-1)}^{k}(-1)^{j} q^{n(3 n-1) / 2}\right)-\sum_{n=0}^{\infty} q^{n(n+1) / 2}\right) \\
& =\left(\sum_{n=0}^{\infty} q^{n(n+1) / 2}\right)\left(\sum_{n=0}^{\infty} M_{k}(n) q^{n}\right),
\end{aligned}
$$


where we have invoked the generating function for $\sigma \operatorname{mex}(n)[5,4]$,

$$
\sum_{n=0}^{\infty} \sigma \operatorname{mex}(n) q^{n}=\frac{\left(q^{2} ; q^{2}\right)_{\infty}}{(q ; q)_{\infty}\left(q ; q^{2}\right)_{\infty}}
$$

and the generating function for $M_{k}(n)[2]$,

$$
\sum_{n=0}^{\infty} M_{k}(n) q^{n}=\sum_{n=k}^{\infty} \frac{q^{\left(\begin{array}{l}
k \\
2
\end{array}\right)+(k+1) n}}{(q ; q)_{n}}\left[\begin{array}{l}
n-1 \\
k-1
\end{array}\right] .
$$

The proof follows easily considering Cauchy's multiplication of two power series.

Combinatorial proof of Theorem 1.3. The statement of Theorem 1.3 is equivalent to identity (2) together with

$$
\begin{aligned}
\sigma \operatorname{mex}\left(n-\frac{k(3 k+1)}{2}\right)-\sigma \operatorname{mex}\left(n-\frac{k(3 k+5)}{2}-1\right) \\
=\sum_{j=0}^{\infty}\left(M_{k}(n-j(j+1) / 2)+M_{k+1}(n-j(j+1) / 2)\right) .
\end{aligned}
$$

Using (1), identity (4) becomes

$$
\begin{gathered}
\sum_{j=0}^{\infty}\left(p\left(n-\frac{j(j+1)}{2}-\frac{k(3 k+1)}{2}\right)-p\left(n-\frac{j(j+1)}{2}-\frac{k(3 k+5)}{2}-1\right)\right) \\
=\sum_{j=0}^{\infty}\left(M_{k}(n-j(j+1) / 2)+M_{k+1}(n-j(j+1) / 2)\right) .
\end{gathered}
$$

Identity (5) was proved combinatorially in [14]. Together with the combinatorial proof of (1), this gives a combinatorial proof of Theorem 1.3.

Next, we give a combinatorial interpretation for $\sum_{j=0}^{\infty} M_{k}(n-j(j+1) / 2)$. First, we introduce some notation. Given an integer $r$, let $\operatorname{sign}(\mathrm{r})$ denote the sign of $r$, i.e.

$$
\operatorname{sign}(\mathrm{r})= \begin{cases}1 & \text { if } r \geqslant 0 \\ -1 & \text { if } r<0\end{cases}
$$

For integers $k, n$ such that $k \geqslant 1$ and $n \geqslant 0$, we denote by $D_{3}^{(k)}(n)$ the number of partitions $\mu$ of $n$ into distinct parts using three colors, $0,1,2$, and satisfying the following conditions:

(i) $\mu$ has exactly $k$ parts of color 2 and, if $k>1$, twice the smallest part of color 2 is greater than largest part of color 2 . 
(ii) Let $r=\ell_{0}(\mu)-\ell_{1}(\mu)$ be the signed difference in the number of parts colored 0 and the number of parts colored 1 in $\mu$. Let $j=|r|-\frac{1}{2}+\operatorname{sign}(\mathrm{r}) \frac{(-1)^{\mathrm{r}}}{2}$. The largest part of color $j(\bmod 2)$ must equal $j$ more that the smallest part of color 2 .

Then, we have the following proposition.

Proposition 3.1. For integers $k, n$ such that $k \geqslant 1$ and $n \geqslant 0$, we have

$$
\sum_{j=0}^{\infty} M_{k}(n-j(j+1) / 2)=D_{3}^{(k)}(n) .
$$

Proof. Take a partition counted by $M_{k}(n-j(j+1) / 2)$ and consider its Ferrers diagram. Remove the first $k$ columns and color the length of each of these columns with color 2. To the remaining Ferrers diagram, add the rotated Ferrers diagram of a staircase $\eta(j)$ of height $j$ and perform the transformation in the combinatorial proof of Theorem 1.1. It is now straight forward that this transformation is a bijection between the sets of partitions counted by the two sides of (6).

Combining Theorems 1.1 and 1.3, and Proposition 3.1 we obtain the following corollary which, by the discussion above, has both analytic and combinatorial proofs.

Corollary 3.2. For integers $k, n$ such that $k \geqslant 1$ and $n \geqslant 0$, we have

$$
(-1)^{\max (0, k-1)}\left(\sum_{j=-\max (0, k-1)}^{k}(-1)^{j} \sigma \operatorname{mex}(n-j(3 j-1) / 2)-\delta(n)\right)=D_{3}^{(k)}(n) .
$$

Note that, if $k=0$, the statement of the corollary reduces to Theorem 1.1.

\section{Connections with overpartitions}

Overpartitions are ordinary partitions with the added condition that the first appearance of any part may be overlined or not. There are eight overpartitions of 3:

$$
3, \overline{3}, 2+1, \overline{2}+1,2+\overline{1}, \overline{2}+\overline{1}, 1+1+1, \overline{1}+1+1 .
$$

In [3], the authors denoted by $\bar{M}_{k}(n)$ the number of overpartitions of $n$ in which the first part larger than $k$ appears at least $k+1$ times. For example, $\bar{M}_{2}(12)=16$, and the partitions in question are $4+4+4, \overline{4}+4+4,3+3+3+3$, $\overline{3}+3+3+3,3+3+3+2+1,3+3+3+\overline{2}+1,3+3+3+2+\overline{1}, 3+3+3+\overline{2}+\overline{1}$, $\overline{3}+3+3+2+1, \overline{3}+3+3+\overline{2}+1, \overline{3}+3+3+2+\overline{1}, \overline{3}+3+3+\overline{2}+\overline{1}$, $3+3+3+1+1+1,3+3+3+\overline{1}+1+1, \overline{3}+3+3+1+1+1, \overline{3}+3+3+\overline{1}+1+1$.

We have the following identity. 
Theorem 4.1. For integers $k, n>0$, we have

$$
\begin{aligned}
& (-1)^{k}\left(\sigma \operatorname{mex}(n)+2 \sum_{j=1}^{k}(-1)^{j} \sigma \operatorname{mex}\left(n-j^{2}\right)-\delta^{\prime}(n)\right) \\
& =\sum_{j=-\infty}^{\infty}(-1)^{j} \bar{M}_{k}(n-j(3 j-1)),
\end{aligned}
$$

where

$$
\delta^{\prime}(n)= \begin{cases}(-1)^{m}, & \text { if } n=m(3 m-1), m \in \mathbb{Z}, \\ 0, & \text { otherwise }\end{cases}
$$

Proof. According to [3, Theorem 7], we have

$$
\begin{aligned}
& \frac{(-q ; q)_{\infty}}{(q ; q)_{\infty}}\left(1+2 \sum_{j=1}^{k}(-1)^{j} q^{j^{2}}\right) \\
& =1+2(-1)^{k} \frac{(-q ; q)_{k}}{(q ; q)_{k}} \sum_{j=0}^{\infty} \frac{q^{(k+1)(k+j+1)}\left(-q^{k+j+2} ; q\right)_{\infty}}{\left(1-q^{k+j+1}\right)\left(q^{k+j+2} ; q\right)_{\infty}}
\end{aligned}
$$

where

$$
\sum_{n=0}^{\infty} \bar{M}_{k}(n) q^{n}=2 \frac{(-q ; q)_{k}}{(q ; q)_{k}} \sum_{j=0}^{\infty} \frac{q^{(k+1)(k+j+1)}\left(-q^{k+j+2} ; q\right)_{\infty}}{\left(1-q^{k+j+1}\right)\left(q^{k+j+2} ; q\right)_{\infty}} .
$$

Multiplying both sides of (7) by

$$
\left(q^{2}, q^{2}\right)_{\infty}=\sum_{n=-\infty}^{\infty}(-1)^{n} q^{n(3 n-1)}
$$

we obtain

$$
\begin{aligned}
& (-1)^{k}\left(\left(\sum_{n=0}^{\infty} \sigma \operatorname{mex}(n) q^{n}\right)\left(1+2 \sum_{j=1}^{k}(-1)^{j} q^{j^{2}}\right)-\sum_{n=-\infty}^{\infty}(-1)^{n} q^{n(3 n-1)}\right) \\
& =\left(\sum_{n=-\infty}^{\infty}(-1)^{n} q^{n(3 n-1)}\right)\left(\sum_{n=0}^{\infty} \bar{M}_{k}(n) q^{n}\right)
\end{aligned}
$$

and the proof follows easily.

Related to Theorem 4.1, we remark that there is a substantial amount of numerical evidence to conjecture the following inequality.

Conjecture 1. For $k, n>0$,

$$
\sum_{j=-\infty}^{\infty}(-1)^{j} \bar{M}_{k}(n-j(3 j-1)) \geqslant 0,
$$

with strict inequality if $n \geqslant(k+1)^{2}$. 
It would be very appealing to have a combinatorial interpretation for the sum in this conjecture.

\section{Connections with partitions into distinct parts}

Following the notation for the number of partitions of $n$ into distinct parts of two colors, we denote by $D_{1}(n)$ the number of partitions of $n$ into distinct parts. We prove the following identity.

Theorem 5.1. For any integer $n \geqslant 0$, we have

$$
\sum_{j=0}^{\infty}(-1)^{j(j+1) / 2} \sigma \operatorname{mex}(n-j(j+1) / 2)=\sum_{j=0}^{\infty} D_{1}\left(\frac{n-j(j+1) / 2}{2}\right),
$$

where $D_{1}(x)=0$ if $x$ is not a positive integer.

Proof. Considering the classical theta identity [1, p. 23, eq. (2.2.13)]

$$
\frac{\left(q^{2} ; q^{2}\right)_{\infty}}{\left(-q ; q^{2}\right)_{\infty}}=\sum_{n=0}^{\infty}(-q)^{n(n+1) / 2}
$$

we can write

$$
\begin{aligned}
\left(\sum_{n=0}^{\infty} \sigma \operatorname{mex}(n) q^{n}\right)\left(\sum_{n=0}^{\infty}(-q)^{n(n+1) / 2}\right) & =\frac{\left(q^{2} ; q^{2}\right)_{\infty}}{(q ; q)_{\infty}\left(q ; q^{2}\right)_{\infty}} \cdot \frac{\left(q^{2} ; q^{2}\right)_{\infty}}{\left(-q ; q^{2}\right)_{\infty}} \\
& =\left(-q^{2} ; q^{2}\right)_{\infty} \cdot \frac{\left(q^{2} ; q^{2}\right)_{\infty}}{\left(q ; q^{2}\right)_{\infty}} \\
& =\left(\sum_{n=0}^{\infty} D_{1}(n) q^{2 n}\right)\left(\sum_{n=0}^{\infty} q^{n(n+1) / 2}\right)
\end{aligned}
$$

and the proof follows by equating the coefficients of $q^{n}$ in this identity.

To obtain a combinatorial interpretation for the sum on the right hand side of (8), let $D_{2}^{*}(n)$ be the number of partitions of $n$ with distinct parts using two colors such that: (i) parts of color 0 form a gap-free partition (staircase) and (ii) only even parts can have color 1 . Then, we have the following identity of Watson type [6].

Proposition 5.2. For $n \geqslant 0$,

$$
\sum_{j=0}^{\infty} D_{1}\left(\frac{n-j(j+1) / 2}{2}\right)=D_{2}^{*}(n)
$$

Proof. To see this, let $\lambda$ be a partition counted by $D_{1}\left(\frac{n-j(j+1) / 2}{2}\right)$. Double the size of each part of $\lambda$ to obtain a partition $\mu$ of $n-j(j+1) / 2$ whose parts are 
even and distinct. Color the parts of $\mu$ with color 1 and add parts $1,2, \ldots, j$ in color 0 to obtain a partition counted by $D_{2}^{*}(n)$. This transformation is clearly reversible.

In [3], the authors denoted by $M P_{k}(n)$ the number of partitions of $n$ in which the first part larger than $2 k-1$ is odd and appears exactly $k$ times. All other odd parts appear at most once. For example, $M P_{2}(19)=10$, and the partitions in question are $9+9+1,9+5+5,8+5+5+1,7+7+3+2$, $7+7+2+2+1,7+5+5+2,6+5+5+3,6+5+5+2+1,5+5+3+2+2+2$, $5+5+2+2+2+2+1$.

We remark the following truncated form of Theorem 5.1.

Theorem 5.3. For integers $n, k>0$,

$$
\begin{aligned}
& (-1)^{k-1}\left(\sum_{j=0}^{2 k-1}(-1)^{j(j+1) / 2} \sigma \operatorname{mex}(n-j(j+1) / 2)-D_{2}^{*}(n)\right) \\
& =\sum_{j=0}^{n} M P_{k}(j) D_{2}^{*}(n-j) .
\end{aligned}
$$

Proof. According to [3, Theorem 9], we have

$$
\begin{aligned}
& \frac{\left(-q ; q^{2}\right)_{\infty}}{\left(q^{2} ; q^{2}\right)_{\infty}} \sum_{j=0}^{2 k-1}(-q)^{j(j+1) / 2} \\
& \quad=1+(-1)^{k-1} \frac{\left(-q ; q^{2}\right)_{k}}{\left(q^{2} ; q^{2}\right)_{k-1}} \sum_{j=0}^{\infty} \frac{q^{k(2 j+2 k+1)}\left(-q^{2 j+2 k+3} ; q^{2}\right)_{\infty}}{\left(q^{2 k+2 j+2} ; q^{2}\right)_{\infty}}
\end{aligned}
$$

where

$$
\sum_{n=0}^{\infty} M P_{k}(n) q^{n}=\frac{\left(-q ; q^{2}\right)_{k}}{\left(q^{2} ; q^{2}\right)_{k-1}} \sum_{j=0}^{\infty} \frac{q^{k(2 j+2 k+1)}\left(-q^{2 j+2 k+3} ; q^{2}\right)_{\infty}}{\left(q^{2 k+2 j+2} ; q^{2}\right)_{\infty}} .
$$

The proof follows easily by multiplying both sides of (10) by

$$
\frac{\left(q^{2} ; q^{2}\right)_{\infty}}{(q ; q)_{\infty}\left(q ; q^{2}\right)_{\infty}} \cdot \frac{\left(q^{2} ; q^{2}\right)_{\infty}}{\left(-q ; q^{2}\right)_{\infty}}
$$

A further interesting corollary of Theorem 5.3 relates to $\sigma \operatorname{mex}(n)$.

Corollary 5.4. For integers $n, k>0$,

$$
(-1)^{k-1}\left(\sum_{j=0}^{2 k-1}(-1)^{j(j+1) / 2} \sigma \operatorname{mex}(n-j(j+1) / 2)-D_{2}^{*}(n)\right) \geqslant 0,
$$

with strict inequality if $n \geqslant k(2 k+1)$. 
On the other hand, the reciprocal of the infinite product in (9) is the generating function for $\operatorname{pod}(n)$, the number of partitions of $n$ in which odd parts are not repeated, i.e.,

$$
\frac{\left(-q ; q^{2}\right)_{\infty}}{\left(q^{2} ; q^{2}\right)_{\infty}}=\sum_{n=0}^{\infty} \operatorname{pod}(n) q^{n}
$$

The properties of the partition function $\operatorname{pod}(n)$ were studied in [10] by Hirschhorn and Sellers. We easily deduce the following convolution identity.

Corollary 5.5. For $n \geqslant 0$,

$$
\sigma \operatorname{mex}(n)=\sum_{j=0}^{n} \operatorname{pod}(j) D_{2}^{*}(n-j)
$$

Finally, we remark that finding a combinatorial interpretation for

$$
\sum_{j=0}^{n} M P_{k}(j) D_{2}^{*}(n-j)
$$

would be very desirable.

\section{Concluding remarks}

The present work began with the search for a combinatorial proof of Theorem 1.1. We were further able to prove several truncated series formulas involving the function $\sigma$ mex. In [5], we worked with the generalization of this function: the sum, $S_{r}(n)$, of $r$-gaps in all partitions of $n$. To keep notation uniform, we use $\sigma \operatorname{mex}_{r}(n)$ for $S_{r}(n)$. Recall that the $r$-gap of a partition $\lambda$ is the least positive integer that does not appear at least $r$ times as a part of $\lambda$. In [5], we proved combinatorially that

$$
\sigma \operatorname{mex}_{r}(n)=\sum_{j \geqslant 0} p(n-r j(j+1) / 2)
$$

and we gave the generating function for $\sigma_{r} \operatorname{mex}(n)$, namely

$$
\sum_{n \geq 0} \sigma_{r} \operatorname{mex}(n) q^{n}=\frac{\left(q^{2 r} ; q^{2 r}\right)_{\infty}}{(q ; q)_{\infty}\left(q^{r} ; q^{2 r}\right)_{\infty}} .
$$

Denote by $\widetilde{D}_{2}^{(r)}(n)$ the number of partitions $\lambda$ of $n$ using two colors, 0 and 1 , such that:

(i) $\lambda^{(0)}$ is a partition into distinct parts divisible by $r$.

(ii) $\lambda^{(1)}$ is a partition with parts repeated at most $2 r-1$ times.

The following generalization of Theorem 1.1 is immediate from (13). 
Theorem 6.1. Let $n, r$ be integers with $r>0$ and $n \geq 0$. Then $\sigma_{r} \operatorname{mex}(n)=$ $\widetilde{D}_{2}^{(r)}(n)$.

Combinatorial proof of Theorem 6.1. Let $\widetilde{\mathcal{D}}_{2}^{(r)}(n)$ be the set of partitions of $n$ counted by $\widetilde{D}_{2}^{(r)}(n)$ described above. Let $\mathcal{P}_{r}(n)$ be the set of partitions of $n$ in which all parts are divisible by $r$. Let $\overline{\mathcal{P}}_{r}(n)$ be the set of partitions of $n$ in which all parts are not divisible by $r$. Finally, let $\mathcal{Q}_{r}(n)$ be the set of partitions of $n$ with parts repeated at most $r-1$ times.

Let $\psi$ denote Glaisher's bijection from $\overline{\mathcal{P}}_{r}(n)$ to $\mathcal{Q}_{r}(n)$.

We create a bijection

$$
\xi: \bigcup_{j \geqslant 0} \mathcal{P}(n-r j(j+1) / 2) \rightarrow \widetilde{\mathcal{D}}_{2}^{(r)}(n) .
$$

Start with a partition $\lambda \in \mathcal{P}(n-r j(j+1) / 2)$ for some $j \geqslant 0$. Let $\tilde{\lambda}$ be the partition consisting of the parts of $\lambda$ that are divisible by $r$ and $\bar{\lambda}$ be the partition consisting of the remaining parts of $\lambda$. Thus all parts of $\bar{\lambda}$ are not divisible by $r$.

Let $\tilde{\lambda}_{/ r}$ be the partition obtained from $\tilde{\lambda}$ by dividing each part by $r$. To $\tilde{\lambda}_{/ r} \in \mathcal{P}\left(\frac{n-|\bar{\lambda}|}{r}-\frac{j(j+1)}{2}\right)$ we apply the bijection $\varphi$ from the combinatorial proof of Theorem 1.1 in section 2. (The appended rotated staircase is $\eta(j)$.) Then $\varphi\left(\tilde{\lambda}_{/ r}\right) \in \mathcal{D}_{2}\left(\frac{n-|\bar{\lambda}|}{r}\right)$. In $\varphi\left(\tilde{\lambda}_{/ r}\right)$, multiply each part of color 0 by $r$ and repeat each part of color 1 exactly $r$ times. These parts, together with the parts of $\psi(\bar{\lambda})$ colored 1, form the partition $\xi(\lambda) \in \widetilde{\mathcal{D}}_{2}^{(r)}(n)$.

Conversely, let $\mu \in \widetilde{\mathcal{D}}_{2}^{(r)}(n)$. Then $\mu^{(0)}$ is a partition with distinct parts all of which are multiples of $r$ and $\mu^{(1)}$ is a partition with parts repeated at most $2 r-1$ times. We write $\mu^{(1)}$ as $\mu^{(1)}=\alpha^{(1)} \cup \beta^{(1)}$, where all parts of $\alpha^{(1)}$ have multiplicity exactly $r$ and all parts of $\beta^{(1)}$ have multiplicity at most $r-1$. Then $\psi^{-1}\left(\beta^{(1)}\right)$ has no part divisible by $r$. We have $\left|\mu^{(0)}\right|=r t_{1},\left|\alpha^{(1)}\right|=r t_{2}$ and $\left|\beta^{(1)}\right|=n-r t_{1}-r t_{2}$ for some non-negative integers $t_{1}$ and $t_{2}$.

Let $\mu_{/ r}^{(0)}$ be the partition with parts colored 0 obtained from $\mu^{(0)}$ by dividing each part by $r$. Then, $\mu_{/ r}^{(0)}$ is a partition with distinct parts colored 0 . Let $\alpha_{\backslash r}^{(1)}$ be the partition with distinct parts colored 1 with exactly the same set of parts as $\alpha^{(1)}$. We then apply $\varphi^{-1}$ to $\mu_{/ r}^{(0)} \cup \alpha_{\downarrow_{r}}^{(1)} \in \mathcal{D}_{2}\left(t_{1}+t_{2}\right)$ to obtain $\varphi^{-1}\left(\mu_{/ r}^{(0)} \cup \alpha_{\backslash r}^{(1)}\right) \in \mathcal{P}\left(t_{1}+t_{2}-\frac{j(j+1)}{2}\right)$ for some non-negative integer $j$. We multiply each part of $\varphi^{-1}\left(\mu_{/ r}^{(0)} \cup \alpha_{\backslash r}^{(1)}\right)$ by $r$. These parts, together with the parts of $\psi^{-1}\left(\beta^{(1)}\right)$, form the partition $\xi^{-1}(\mu) \in \mathcal{P}(n-r j(j+1) / 2)$.

Example 6.1. Let $n=167, r=3, j=3$ and consider $\lambda=21+21+19+18+18+12+8+8+8+8+6+1+1 \in \mathcal{P}(167-3 \cdot 6)=\mathcal{P}(149)$. 
Then,

$$
\begin{aligned}
& \tilde{\lambda}=21+21+18+18+12+6 \in \mathcal{P}_{3}(96), \\
& \bar{\lambda}=19+8+8+8+8+1+1 \in \overline{\mathcal{P}}_{3}(53),
\end{aligned}
$$

and

$$
\tilde{\lambda}_{/ 3}=7+7+6+6+4+2 \in \mathcal{P}(32) .
$$

Applying Glaisher's bijection, we have $\psi(\bar{\lambda})=24+19+8+1+1 \in \mathcal{Q}_{3}(53)$. From Example 2.3 , we have $\varphi\left(\tilde{\lambda}_{/ 3}\right)=9_{1}+8_{1}+6_{1}+5_{1}+3_{1}+3_{0}+2_{1}+2_{0}$. Now, we multiply parts of color 0 by 3 , repeat each part of color 1 three times, and include the parts of $\psi(\bar{\lambda})$ with color 1 to obtain

$$
\begin{aligned}
\xi(\lambda)= & 24_{1}+19_{1}+9_{1}+9_{1}+9_{1}+9_{0}+8_{1}+8_{1}+8_{1}+8_{1}+6_{1}+6_{1}+6_{1}+ \\
& 6_{0}+5_{1}+5_{1}+5_{1}+3_{1}+3_{1}+3_{1}+2_{1}+2_{1}+2_{1}+1_{1}+1_{1} \in \widetilde{\mathcal{D}}_{2}^{(3)}(167) .
\end{aligned}
$$

Conversely, let

$$
\begin{aligned}
\mu= & 24_{1}+19_{1}+9_{1}+9_{1}+9_{1}+9_{0}+8_{1}+8_{1}+8_{1}+8_{1}+6_{1}+6_{1}+6_{1}+ \\
& 6_{0}+5_{1}+5_{1}+5_{1}+3_{1}+3_{1}+3_{1}+2_{1}+2_{1}+2_{1}+1_{1}+1_{1} \in \widetilde{\mathcal{D}}_{2}^{(3)}(167) .
\end{aligned}
$$

Then, we have the following relevant partitions.

$$
\begin{aligned}
\mu^{(0)}= & 9_{0}+6_{0} \in \mathcal{P}_{3}(15) \\
\mu^{(1)}= & 24_{1}+19_{1}+9_{1}+9_{1}+9_{1}+8_{1}+8_{1}+8_{1}+8_{1}+6_{1}+6_{1}+6_{1}+ \\
& 5_{1}+5_{1}+5_{1}+3_{1}+3_{1}+3_{1}+2_{1}+2_{1}+2_{1}+1_{1}+1_{1} \in \mathcal{Q}_{6}(152) \\
\alpha^{(1)}= & 9_{1}+9_{1}+9_{1}+8_{1}+8_{1}+8_{1}+6_{1}+6_{1}+6_{1}+5_{1}+5_{1}+5_{1}+ \\
& 3_{1}+3_{1}+3_{1}+2_{1}+2_{1}+2_{1} \in \mathcal{P}(99) \\
\beta^{(1)}= & 24_{1}+19_{1}+8_{1}+1_{1}+1_{1} \in \mathcal{Q}_{3}(53) \\
\psi^{-1}\left(\beta^{(1)}\right)= & 19_{1}+8_{1}+8_{1}+8_{1}+8_{1}+1_{1}+1_{1} \in \overline{\mathcal{P}}_{3}(53) \\
\mu_{/ 3}^{(0)}= & 3_{0}+2_{0} \\
\alpha_{\backslash 3}^{(1)}= & 9_{1}+8_{1}+6_{1}+5_{1}+3_{1}+2_{1}
\end{aligned}
$$

Then $\mu_{/ 3}^{(0)} \cup \alpha_{\backslash 3}^{(1)} \in \mathcal{D}_{2}(38)$ and from Example 2.3, we have $j=3$ and

$$
\varphi^{-1}\left(\mu_{/ 3}^{(0)} \cup \alpha_{\backslash 3}^{(1)}\right)=7+7+6+6+4+2 \in \mathcal{P}(32)=\mathcal{P}\left(38-\frac{3(3+1)}{2}\right) .
$$

Now we multiply all parts of $\varphi^{-1}\left(\mu_{/ 3}^{(0)} \cup \alpha_{\backslash_{3}}^{(1)}\right)$ by 3 and include the parts of $\psi^{-1}\left(\beta^{(1)}\right)$ with the color removed to obtain

$\xi^{-1}(\mu)=21+21+19+18+18+12+8+8+8+8+6+1+1 \in \mathcal{P}(149)=\mathcal{P}(167-3 \cdot 6)$. 


\section{References}

[1] G.E. Andrews, The Theory of Partitions, Cambridge Math. Lib., Cambridge University Press, Cambridge, 1998.

[2] G.E. Andrews, M. Merca, The truncated pentagonal number theorem, J. Combin. Theory Ser. A, 119 (2012) 1639-1643.

[3] G.E. Andrews, M. Merca, Truncated Theta Series and a Problem of Guo and Zeng, J. Combin. Theory Ser. A, 154 (2018) 610-619.

[4] G.E. Andrews, D. Newman, Partitions and the minimal excludant, Ann. Comb., 23(2) (2019) 249-254.

[5] C. Ballantine, M. Merca, Bisected theta series, least $r$-gaps in partitions, and polygonal numbers Ramanujan J, 52 (2020) 433-444.

[6] C. Ballantine, M. Merca, On identities of Watson type, Ars Math. Contemp., 17 (2019), no. 1, 277-290.

[7] S. Corteel, J. Lovejoy, Overpartitions, Trans. Amer. Math. Soc., 356 (2004) $1623-1635$.

[8] P. J. Grabner, A. Knopfmacher, Analysis of some new partition statistics. Ramanujan J. 12 (2006), no. 3, 439-454.

[9] P.M. Grundy, Mathematics and games, Eureka 2 (1939) 6-8. Reprinted in, Eureka 27 (1964) 9-11.

[10] M.D. Hirschhorn, J.A. Sellers, Arithmetic properties of partitions with odd parts distinct, Ramanujan J, 22 (2010) 273-284.

[11] R.P. Sprague, Über mathematische Kampfspiele, Tohoku Math. J., 41 (193536) 438-444.

[12] J.J. Sylvester, F. Franklin, A constructive theory of partitions, arranged in three acts, an interact and an exodion, Amer. J. Math., 5 (1882) 251-330.

[13] E.M. Wright, An enumerative proof of an identity of Jacobi, J. London Math. Soc., 40 (1965) 55-57.

[14] A.J. Yee, A truncated Jacobi triple product theorem, J. Combin. Theory Ser. A, 130 (2015), 1-14. 